BULL. AUSTRAL. MATH. SOC.

$16 A \mid 8$

VOL. II (1974), 365-371.

\title{
Torsionfree modules and classes of orders
}

\section{William H. Gustafson}

\begin{abstract}
It is shown how torsionfree modules can be used to characterize certain important classes of orders over Dedekind rings. In particular, we show that an order is Gorenstein if and only if each of its lattices can be embedded as a pure sublattice of a free lattice. We also show that an order is hereditary if and only if the tensor product of any of its right lattices with any of its left lattices is torsionfree over the ground domain.
\end{abstract}

\section{Introduction}

Let us gather some notation and definitions. Let $R$ be a Dedekind ring with field of fractions $K$, and let $\Lambda$ be an $R$-order in a semisimple $K$-algebra $A$. That is, $\Lambda$ is an $R$-subalgebra of $A$, containing the identity, such that $\Lambda$ is finitely generated as an $R$-module and contains a $K$-basis for $A$. By a $\Lambda$-lattice, we mean a $\Lambda$-module which is finitely generated and projective as $R$-module. Denote by $\Lambda$-lat (respectively, lat- $\Lambda$ ) the category of left (respectively, right) $\Lambda$-lattices.

We say that the left $\Lambda$-lattice $L$ is weakly injective if $\operatorname{ext}_{\Lambda}^{l}(N, L)=0$ for each left $\Lambda$-lattice $N . \Lambda$ is left Gorenstein if $\Lambda$ is weakly injective as a left $\Lambda$-lattice. By Theorem 3.3 of [1], $\Lambda$ is left Gorenstein if and only if the injective dimension of $\Lambda$ as a left $\Lambda$-module is one (assuming $R \neq K$ ). It follows that our definition of left Gorenstein orders is in accord with the usual one given in [2]. Further,

Received 8 July 1974 . 
Proposition $\dot{6} .1$ of [2] shows that $\Lambda$ is left Gorenstein if and only if it is right Gorenstein in the obvious sense. Hence, we can refer to left Gorenstein orders simply as Gorenstein orders.

Given a $\Lambda$-lattice $M$ and a sublattice $N$, we say that $N$ is a pure sublattice of $M$ if $M / N$ is R-torsionfree. Our characterization of Gorenstein orders is the following theorem.

THEOREM 1. The R-order $\Lambda$ is Gorenstein if and only if each left $\Lambda$-lattice is isomorphic to a pure sublattice of a free left $\Lambda$-lattice.

The $R$-order $\Lambda$ is said to be hereditary if it is an hereditary ring. It follows from the semisimplicity of $A$ that any $\Lambda$-lattice can be embedded in a free $\Lambda$-lattice, hence $\Lambda$ is hereditary if and only if all its lattices are projective $\Lambda$-modules. In particular, an hereditary order is Gorenstein. Hattori [4] showed that a noetherian integral domain $R$ is Dedekind if and only if the tensor product of any two torsionfree $R$-modules is again torsionfree. Our characterization of hereditary orders is very similar to this theorem.

THEOREM 2. The R-order $\Lambda$ is hereditary if and only if $M \otimes_{\Lambda} N$ is $R$-torsionfree whenever $M \in l$ at $\Lambda$ and $N \in \Lambda$-lat.

\section{Gorenstein orders}

In this section we prove Theorem $I$ and discuss some related results. It is clear that the theorem is analogous to the familiar result that a finite dimensional algebra over a field is quasi-Frobenius if and only if each of its finitely generated modules is torsionless. (As we have noted above, each lattice over an order is torsionless, hence the additional requirement of purity in Theorem 1 plays an indispensable role.) By analogy, it is natural to prove the theorem using the dualizing functor $\operatorname{hom}_{R}(-, R)$, which sends $\Lambda$-lat to lat $\Lambda$, and conversely. As is customary, we shall denote $\operatorname{hom}_{R}(M, R)$ by $M^{*}$. Then, we have the natural isomorphism $M \cong M^{* *}$ for lattices. Further, $\operatorname{hom}_{R}(-, R)$ is an exact functor on the categories of lattices. It is not difficult to show that $M$ is projective if and only if $M^{*}$ is weakly injective. 
Let us now proceed to the proof of Theorem 1. Assume first that $\Lambda$ is Gorenstein and let $M$ be a left $\Lambda$-lattice. Find an exact sequence

$$
0 \rightarrow N \rightarrow F \rightarrow M^{*} \rightarrow 0 \text {, }
$$

where $F$ is a free right $\Lambda$-lattice. By duality, we obtain the exact sequence

$$
0 \rightarrow M \rightarrow E^{*} \rightarrow N^{*} \rightarrow 0 \text {. }
$$

Since $\Lambda$ is Gorenstein, $F^{*}$ is projective. Further, $N^{*}$ is a lattice, hence $R$-torsionfree. Thus, $M$ is a pure sublattice of the projective lattice $F^{*}$. If necessary, we may form the direct sum of $F^{*}$ and another $\Lambda$-lattice so as to obtain a free $\Lambda$-lattice in which $M$ is embedded as a pure sublattice.

Conversely, assume that each left $\Lambda$-lattice can be embedded as a pure sublattice of a free lattice. Embed $\left(\Lambda_{\Lambda}\right) *$ as a pure sublattice of a free $\Lambda$-lattice $F$. Thus, we have an exact sequence

$$
0 \rightarrow\left(\Lambda_{\Lambda}\right) *+F \rightarrow X \rightarrow 0
$$

where $X$ is a $\Lambda$-lattice. By duality, we obtain the exact sequence

$$
0 \rightarrow X^{*} \rightarrow F^{*} \rightarrow \Lambda_{\Lambda} \rightarrow 0
$$

Since $\Lambda_{\Lambda}$ is free, this sequence splits. Therefore, $\Lambda_{\Lambda}$ is a direct summand of the weakly injective lattice $F^{*}$, and is hence itself weakly injective. Thus $\Lambda$ is Gorenstein and the proof of Theorem $I$ is complete.

We wish now to indicate a possible method of applying this theorem to the study of orders of finite representation type, that is, those which have only finitely many isomorphism types of indecomposable lattices.

COROLLARY. Assume that the Jordan-Zassenhaus Theorem [9] is valid for $R$-orders, and let $\Lambda$ be an R-order. Then $\Lambda$ is a corenstein order of finite representation type if and only if $\Lambda$ is Morita-equivalent to an order $\Gamma$ with the property that each indecomposable $\Gamma$-lattice is isomorphic to a pure ideal of $\Gamma$.

Proof. If $\Lambda$ is Morita-equivalent to such an order $\Gamma$, then $\Gamma$ has only finitely many indecomposable lattices by Jordan-Zassenhaus, and $\Gamma$ is Gorenstein by the theorem. Since both these properties are Norita- 
invariant, $\Lambda$ also enjoys them.

On the other hand, let $\Lambda$ be Gorenstein and have only finitely many indecomposable lattices. Then there is a free $\Lambda$-module $F$ in which all the indecomposable $\Lambda$-lattices can be embedded as pure sublattices. It is now easy to verify that $\Gamma=\operatorname{hom}_{\Lambda}(F, F)^{\circ p}$ has the property that each of its indecomposable lattices is isomorphic to a pure ideal. This completes the proof.

We remark that it may indeed be necessary to effect a proper Morita equivalence to obtain the order $\Gamma$ in the above corollary. For example, let $p \geq 3$ be a rational prime, $R$ the ring of $p$-adic integers and $G$ a cyclic group of order $p^{2}$. Then the group ring $R G$ is a Gorenstein order with only finitely many indecomposable lattices. However, some of these indecomposable $\Lambda$-lattices are of $R$-rank $p^{2}+1$, and are therefore not isomorphic to ideals of $R G$. The indecomposable $R G$-lattices are listed in [5] and [6].

Finally, let us remark that by using the main result of [7], one can imitate the proof of the above corollary to show

PROPOSITION. Let $A$ be a finite-dimensional K-algebra. Then $A$ is a quasi-Frobenius algebra of finite representation type if and only if $A$ is Morita-equivalent to an algebra $B$, all of whose indecomposable modules are isomorphic to ideals.

Once again, it is possible to give examples showing that a proper Morita-equivalence may be needed. One such example is presented in [3].

\section{Hereditary orders}

In this section we give a proof of Theorem 2, along the lines of the work of Hattori mentioned in the introduction.

Let us first suppose that $\Lambda$ is hereditary, $M \in$ lat $-\Lambda$ and $N \in \Lambda$-lat . Since every $\Lambda$-lattice is projective, we may find $X \in \Lambda$-lat such that $N \oplus X$ is a free $\Lambda$-lattice. Then $M \Theta_{\Lambda} N$ is an $R$-direct summand of $M \otimes_{\Lambda}(N \oplus X)$. The latter module is $R$-isomorphic to a direct sum of copies of $M$, hence $M \otimes_{\Lambda} N$ is $R$-torsionfree. 
Conversely, suppose that $\Lambda$ is not hereditary. Then there is a nonprojective right $\Lambda$-lattice $M$. It follows from $[8, \mathrm{p} .62]$ that $M$ is not a flat $\Lambda$-module. Hence, there is a left $\Lambda$-module $X$ such that $\operatorname{tor}_{1}^{\Lambda}(M, X) \neq 0$. Since $\operatorname{tor}_{1}^{\Lambda}(M,-)$ commutes with direct limits, we may assume that $X$ is finitely generated. We now find an exact sequence

$$
0 \rightarrow N \rightarrow F+X \rightarrow 0 \text {, }
$$

where $F$ is a finitely generated free left $\Lambda$-lattice. Applying $M \otimes_{\Lambda}-$ to this sequence, we obtain the exact sequence

$$
0 \rightarrow \operatorname{tor}_{\perp}^{\Lambda}(M, X) \rightarrow M \otimes_{\Lambda} N \rightarrow M \otimes_{\Lambda} F \rightarrow M \otimes_{\Lambda} X \rightarrow 0,
$$

whence $M \otimes_{\Lambda} N$ contains an $R$-submodule isomorphic to $\operatorname{tor}_{1}(M, X)$. The proof is now completed by appealing to the next proposition.

PROPOSITION. Let $M$ be a right $\Lambda$-module and $X$ a left $\Lambda$-module. Then $\operatorname{tor}_{n}^{\Lambda}(M, X)$ is a torsion R-module for all $n \geq 1$.

Proof. We give a proof in four steps.

Step I. By a direct limit argument, we see that we may assume that $X$ is finitely generated.

Step II. Assume that $X$ is a torsion $R$-module. Then since $X$ is finitely generated as $R$-module, there is a nonzero $\alpha \in R$ such that $\alpha X=0$. It follows that $\operatorname{\alpha tor}_{n}^{\Lambda}(M, X)=0$ for all $n \geq 0$.

Step III. Assume that $X$ is torsionfree as an $R$-module, so that $X$ is a $\Lambda$-lattice. Because $A$ is semisimple, we can find another $\Lambda$-lattice $Y$ and a free $\Lambda$-lattice $F$ such that $X \oplus Y \subseteq F$ and $F /(X \oplus Y)$ is a torsion $R$-module. The exact homology sequence yields an isomorphism

$$
\operatorname{tor}_{n+1}^{\Lambda}(M, F /(X \oplus Y)) \cong \operatorname{tor}_{n}^{\Lambda}(M, X) \oplus \operatorname{tor}_{n}^{\Lambda}(M, Y) \text {. }
$$

By Step II, $\operatorname{tor}_{n}^{\Lambda}(M, X)$ is torsion.

Step IV. Now we deal with an arbitrary finitely generated $X$. Let $t X$ be the $R$-torsion submodule of $X$; clearly, it is a $\Lambda$-submodule. From the exact sequence 


$$
0 \rightarrow t X \rightarrow X \rightarrow X / t X \rightarrow 0
$$

we obtain an exact sequence

$$
\operatorname{tor}_{n}^{\Lambda}(M, t X) \rightarrow \operatorname{tor}_{n}^{\Lambda}(M, X) \rightarrow \operatorname{tor}_{n}^{\Lambda}(M, X / t X) .
$$

Since the end terms are $R$-torsion, so is the middle term. This completes the proof.

\section{References}

[1] Hyman Bass, "Injective dimension in Noetherian rings", Trons. Amer. Math. Soc. 102 (1962), 18-29.

[2] Ю. А. Дрозд, В.В. Ннриченно, А.В. Ройтер [Ju.A. Drozd, V.V. Ki ričcenko, A.V. RoITter], "О наследственных н бассовых поряднах" [On hereditary and Bass orders], Izv. Akad. Nauk SSSR Ser. Mat. 31 (1967), 1415-1436; English translation: Math. USSR Izv. 1 (1967), 1357-1376.

[3] E. Green and W. Gustafson, "Pathological quasi-Frobenius algebras of finite type", Comm. Algebra (to appear).

[4] Akira Hattori, "On Prüfer rings", J. Math. Soc. Jcopan 9 (1957), 381-385.

[5] A. Heller and 1. Reiner, "Representations of cyclic groups in rings of integers I", Ann. of Math. (2) 76 (1962), 73-92.

[6] А.В. Яновлев [A. Jakovlev], "Гомологичесная определенность $p$-аднчесних представлений нолец со степенным базисом" [Homological determination of p-adic representations of rings with power basis], Izv. Akad. Nauk SSSR Ser. Mat. 34 (1970), 1000-1014; English translation: Math. USSR Iav. 4 (1970), 1001-1016. 
[1] A.B. Ройтер [A.V. RoĬter], "Неограниченность размерностей неразлонимых представлений алгебры, именацей беснонечно много неразлонимых представлений" [Unboundedness of the dimensions of indecomposable representations of algebras having infinitely many indecomposable representations], Izv. Akad. Nauk SSSR Ser. Mat. 32 (1968), 1275-1282; English translation: Math. USSR Izv. 2 (1968), $1223-1230$.

[8] Joseph J. Rotman, Notes on homological algebra (Van Nostrand Reinhold Mathematical Studies, 27. Van Nostrand Reinhold Company, London, New York, Cincinnati, Toronto, Melbourne, 1970).

[9] Richard E. Swan, K-theory of finite groups and orders (notes by $E$. Graham Evans. Lecture Notes in Mathematics, 149. SpringerVerlag, Berlin, Heidelberg, New York, 1970).

Department of Mathematics, Indiana University, Indiana, USA. 